\title{
Changing Expression of Cyclooxygenases and Prostaglandin Receptor EP4 During Development of the Human Ductus Arteriosus
}

\author{
CORNELIA RHEINLAENDER, SVEN C. T. WEBER, NANETTE SARIOGLU, EVELYN STRAUß, MICHAEL OBLADEN, \\ AND PETRA KOEHNE \\ Departments of Neonatology [C.R., S.C.T.W., E.S., M.O., P.K.], Pedopathology and Placentology [N.S.], Charité Universitätsmedizin \\ Berlin, Campus Virchow Hospital, 13353 Berlin, Germany
}

\begin{abstract}
Programmed proliferative degeneration of the human fetal ductus arteriosus (DA) in preparation for its definite postnatal closure has a large developmental variability and is controlled by several signaling pathways, most prominently by prostaglandin (PG) metabolism. Numerous studies in various mammalian species have shown interspecies and developmental differences in ductal protein expression of cyclooxygenase (COX) isoforms and PG E receptor subtypes (EP1-4). We examined COX1, COX2, and EP4 receptor protein expression immunohistochemically in 57 human fetal autopsy DA specimens of 11-38 wk of gestation. According to their histologic maturity, specimens were classified into four stages using a newly designed maturity score that showed that histologic maturity of the DA was not closely related to gestational age. COX1 expression was found in all DA regions and rose steadily during development. COX2 staining remained weak throughout gestation. EP4 receptor staining increased moderately during gestation and was limited to the intima and media. In conclusion, histologic maturity classification helps to address developmentally regulated processes in the fetal DA. Concerning prostaglandin metabolism our findings are in line with animal studies, which assigned COX1 the predominant role in the DA throughout gestation. EP4 receptor presumably plays a key role for active patency of the human DA in the third trimester.
\end{abstract} (Pediatr Res 60: 270-275, 2006)

$\mathrm{D}$ uring fetal life, the DA is the shunt blood vessel between pulmonary artery and aorta, bypassing pulmonary circulation. Due to its closure after birth, it undergoes a different development compared with the main arteries despite their same origin in branching arteries (1). The DA closure is characterized by three steps: (1) intima thickening and remodeling, (2) perinatal constriction, and (3) definite obliteration. This unique programmed proliferative degeneration starts during the early fetal period, underlies a large developmental variation, and is not strongly correlated with gestational age $(2,3)$. Therefore, delayed postnatal spontaneous closure or persistent patency of the DA warranting medical intervention, which occurs in about $50 \%$ of the premature infants, may only in part be explained by immaturity (4). Until now the key regulators that set off this ductus-specific differentiation pro-

Received February 15, 2006; accepted May 10, 2006

Correspondence: Petra Koehne, M.D., Department of Neonatology, Charité Universitätsmedizin Berlin, Campus Virchow-Klinikum, Augustenburger Platz 1, D-13353 Berlin, Germany; e-mail: petra.koehne@charite.de

This study was supported by a Rahel-Hirsch research grant.

DOI: $10.1203 / 01 . p d r .0000233066 .28496 .7 \mathrm{c}$ gram are not well understood, but clearly several pathways are involved (5).

Prostaglandins, and among them mainly prostaglandin $\mathrm{E}_{2}$ (PGE2) and prostacyclin, regulate the ductal tone during pregnancy. Moreover, changes in prostanoid concentration and ductal responsiveness to prostanoids seem to mediate functional DA closure after birth (6).

COXs catalyze the first committed step in PG synthesis. The two isoforms have a similar, and in its structure, highly conserved catalytic center, but their regulatory domains differ. COX1 is a housekeeping gene product and hence constitutively expressed, whereas COX2 is an inducible form with about 10 -fold higher activity than COX1. COX2 is involved in processes with temporary excessive need for prostanoids, such as inflammation and labor.

The effects exerted on the DA by PGs are mainly mediated through PGE2 receptors, of which four subtypes, EP1-4, have been identified by now. Among them, the EP4 receptor has been shown to be the most potent mediator of PGE2 vasodilatating effect on the DA in fetal life (7-9). It induces relaxation of smooth muscle cells via $\mathrm{G}$ protein-coupling and intracellular cyclic adenosine monophosphate (cAMP) increase (7).

Several studies have been carried out on ductal expression of COX and EP receptors in different animal species such as lamb, baboon, rat, rabbit, mouse, and pig. Based on phylogenetic analysis of the PG receptor subtypes, it has been suggested that the COX pathway may have evolved from PGE2 and an ancestral EP receptor; however, interspecies differences in expression have been found (7). Very few studies have been carried out on human tissue, all of them with a small number of cases and a heterogeneous population (10).

We hypothesized that the key players of PG metabolism, both COX isoforms and the EP4 receptor, can also be found in human fetal DA. Furthermore, we were interested whether the expression of those factors changes during fetal development. Therefore, we investigated maturity-dependent protein expression of COX1, COX2, and EP4 receptor in human DA in a

Abbreviations: COX, cyclooxygenase; DA, ductus arteriosus; EP, prostaglandin E receptor subtypes; PDA, patent ductus arteriosus; PG, prostaglandin; PGE2, prostaglandin $\mathrm{E}_{2}$ 
large cohort of fetal autopsy specimens with gestational ages ranging between 11 and 38 wk.

\section{METHODS}

Human tissue specimens. Autopsy specimens of the DA and the corresponding main arteries from 76 human fetuses and four liveborns were collected over 12 mo at the Charité Department of Pedopathology. Informed parental consent was obtained for autopsy. The study was approved by the local ethics committee. Median gestational age was $21 \mathrm{wk}$ (range, 11-38 wk). Thirty-three fetuses/newborns were female and 47 were male. The main causes of death in the fetuses, besides abortion for medical reasons (51 cases), were amnion infection syndrome or other infections (eight cases) and placental insufficiency or placental ablation (12 cases). Although spontaneous miscarriage predominated in the first trimester, intracervical application of synthetic PGE1 (misoprostol, Cytotec) or PGE2 (dinoproston, Minprostin) analogues was used to terminate pregnancies in the second trimester. In two thirds of the cases, either intrauterine death occurred (five cases) or fetocide (21 cases with $\geq 22$ completed wk of gestation) was performed before induction of labor by PG application.

Among the four liveborns, one infant of 36-wk gestation died on $\mathrm{d} 3$ of life from pulmonary artery embolism, another infant of 36-wk gestation died on d 9 of life of cardiorespiratory failure from sepsis, one infant born after $34 \mathrm{wk}$ of gestation with gastroschisis died after $6 \mathrm{wk}$ of necrotizing enterocolitis, and one infant with a gestational age of $38 \mathrm{wk}$ died after $4 \mathrm{~h}$ due to a complex malformation with cardiac involvement. None of the liveborn infants received treatment with PG synthesis inhibitors for a patent DA (PDA).

To assess the correlation between histologic maturity of the DA and the documented gestational age in autopsy reports all 80 specimens were examined. For determination of COX expression, 23 cases with signs of inflammation, autolytic degradation, or malformation of the main arteries were excluded. A frequency distribution of the clinical and pathologic diagnoses of the 57 included cases is summarized in Table 1 . The fetuses were fixed in buffered $4 \%$ formaldehyde. After dissection and micropreparation under a stereomicroscope the vascular tissue samples of DA and the corresponding main arteries (ascending and descending aorta, aortic isthmus and pulmonary trunk) were paraffin embedded, subsequently cut in 3- to 5- $\mu \mathrm{m}$ sections, and mounted on coated slides (SuperFrost Plus, Langenbrinck Laborbedarf, Emmendingen, Germany).

Determination of ductal maturity. For assessment of histologic ductal maturity, sections were deparaffinized, rehydrated, and stained according to an elastica van Giesen protocol, which revealed black elastic fibers, green nuclei, yellow erythrocytes, a purple cytoplasm, and a pink extracellular matrix range 11-38 wk, see Fig. 1. Sections were evaluated by light microscopy.

A histologic maturity score based on studies by Gittenberger-de Groot et al. (2) and Desligneres and Larroche (3), modified by our observations, was used to subdivide DA maturation into four stages from early fetal life until perinatal closure (Table 2). For each of the nine evaluated histologic criteria, between one to four points were given depending on their degree of occurrence. According to the score, specimens were classified into one of the four maturity stages.

Immunohistochemistry. Sections were deparaffinized in xylol for $10 \mathrm{~min}$ twice, rehydrated in graded alcohols $(100,90,80$, and $70 \%)$ and aqua

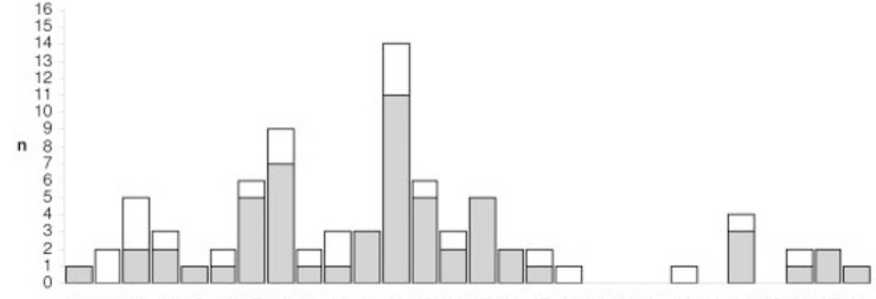

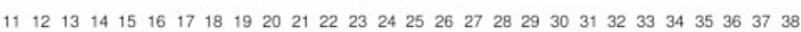

gestational age (completed weeks)

Figure 1. Gestational age-dependent case distribution. Gray bars show the number of cases included for the evaluation of COX1, COX2, and EP4 receptor protein expression.

destillata, for 3 min each at room temperature. COX1 staining was carried out after slight modification of a published protocol (11); for COX1 staining, sections were washed with TBS-Tween $0.05 \%$ for $5 \mathrm{~min}$. Subsequently, sections were incubated overnight at $4{ }^{\circ} \mathrm{C}$ with a monoclonal mouse antihuman COX1 primary antibody (Zymed Laboratories, San Francisco, CA) diluted 1:300 in an antibody diluent (Zymed Laboratories). Using the streptavidin-biotin method in conjunction with an alkaline phosphatase-fast red reaction for detection led to bright red staining of positive cells and was performed with the Super Sensitive Detection System (MultiLink, BioGenex, Laboratories Inc., San Ramon, CA) according to the manufacturer's instructions. TBS-Tween $0.05 \%$ was used as washing buffer after each step. Sections were lightly counterstained with hematoxylin and mounted with aqueous mounting media (Ultramount, DakoCytomation GmbH, Hamburg, Germany).

For COX2 staining, antigen retrieval was performed by cooking sections in citrate buffer $(\mathrm{pH} 6.0)$ at $600 \mathrm{~W}$ for $10 \mathrm{~min}$ in a microwave oven. After cooling, sections were washed in TBS-Tween $0.05 \%$ for $5 \mathrm{~min}$, followed by incubation with the primary mouse anti-human COX2 antibody (Zymed Laboratories) at room temperature for $45 \mathrm{~min}$, diluted 1:500 in phosphatebuffered saline/bovine serum albumin (PBS/BSA) $0.05 \%$. Staining was performed with the catalyzed signal amplification method (CSA-kit, DakoCytomation $\mathrm{GmbH}$ ) according to the manufacturer's instructions. This streptavidinbiotin-based detection system, which uses an additional amplification step with thyramin besides a horseradish peroxidase and 3,3'-diaminobenzidine visualization system, resulted in dark brown staining of positive cells. TBSTween $0.05 \%$ was used as washing buffer after each step. Sections were lightly counterstained with hematoxylin, dehydrated with graded alcohols and xylol, and mounted with a xylol based Neo-Mount mounting media (Merck KG, Darmstadt, Germany).

For EP4 receptor staining, sections were permeabilized for $40 \mathrm{~min}$ with a TBS-Tween $0.05 \%$ /Triton X-100 $0.1 \%$ solution and thereafter washed with PBS. The primary rabbit anti-human polyclonal EP4 receptor antibody (Cayman Chemical Company, Ann Arbor, MI) was diluted 1:200 in PBS/BSA $0.05 \%$ and sections incubated overnight at $4^{\circ} \mathrm{C}$. For detection, a secondary fluorescent goat anti-rabbit antibody (Alexa Fluor 488, MoBiTec GmbH, Goettingen, Germany) was applied at a dilution of 1:2000 in PBS and incubated at room temperature for $1 \mathrm{~h}$. After washing with PBS, slides were

Table 1. Summary of clinical and pathological data from autopsy reports

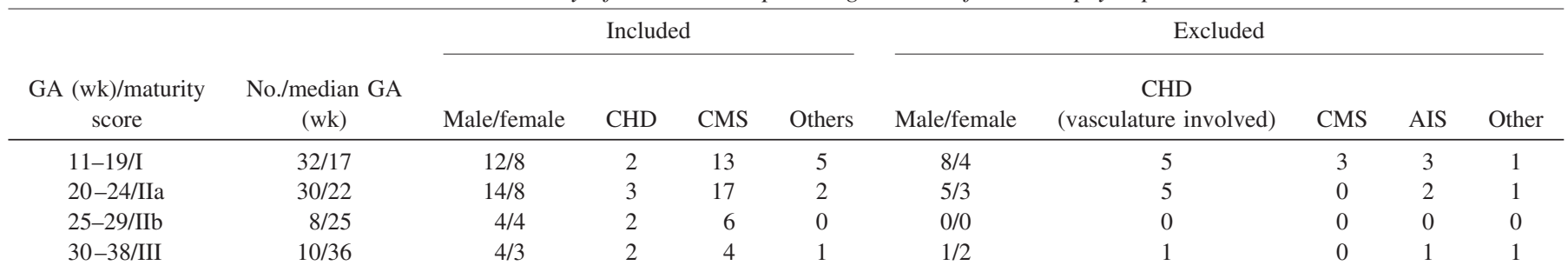

Cases are divided into those included for immunohistochemical examination of COX1, COX2, and EP4 receptor expression and those excluded. Cases with multiple diagnoses not compatible with one of the above-mentioned categories are summarized under "others." Pathologic diagnoses in the inclusion group summarized as CHD included Down syndrome with ventricular septal defect (two cases), hypoplasic left heart syndrome (two cases), aortic valve stenosis (two cases), pulmonary valve atresia (one case), lung hypoplasia combined with ventricular septal defect (one case), and a mosaicism of trisomy 13 with ventricular septal defect. In none of the included cases were heart defects involving the main arteries. Pathologic diagnoses in the inclusion group summarized as CMS included Potter syndrome (four cases), Down syndrome without cardiac malformation (three cases), neural tube defects (five cases), central nervous system malformations (12 cases), skeletal malformations (six cases), chromosomal aberrations without cardiac involvement (five cases), diaphragmatic hernia (two cases), omphalocele (two cases), Pierre Robin sequence (one case). AIS, amnion infection syndrome; CHD, congenital heart defect; CMS, other congenital malformation syndrome; GA, gestational age. 
Table 2. Maturity score for the developing human DA, combining the basic histologic findings of Deligneres and Larroche and Gittenberger-de Groot et al. with our own observations.

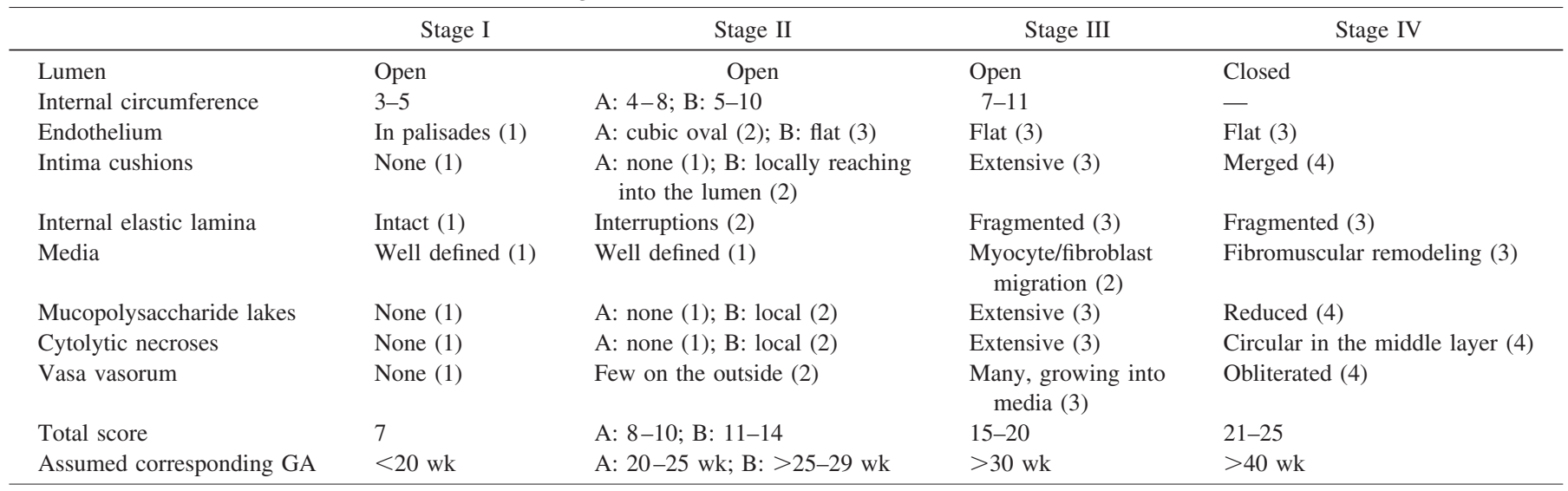

One to four points (numbers in parentheses) are given in each category. Specimens are classified in one of the four maturity grades according to their score of points. GA, gestational age.

counterstained and mounted with an aqueous 4',6-diamidino-2-phenylindole containing fluorescence protecting mounting media (Vectashield, Vector Laboratories, Burlingame, CA).

Human umbilical cord sections were used as methodical positive controls in every experiment. For negative controls, the primary antibody was replaced by a preimmune mouse or rabbit serum, respectively.

Morphometric analysis. COX1 and COX2 staining intensity was evaluated by light microscopy, whereas evaluation of EP4 receptor staining was performed by fluorescence microscopy. Immunohistochemical staining was evaluated semiquantitatively at high-power magnification. On a discrete scale with 0.5 -intervals, points were given from 0 for no staining to 3 for strong staining. The staining intensity was separately scored for three vessel regions: (1) endothelium, (2) subendothelial intima, and (3) media. Histologic classification and immunohistochemical staining were independently rated by three blinded observers (C.R., S.C.T.W., and P.K.). The interobserver difference was $7.1 \%$. Different results were discussed afterward until consensus was reached. For statistical evaluation, the Mann-Whitney $U$ test was applied, and $p<0.05$ was considered significant. Pearson correlation coefficients $\left(R^{2}\right)$ were calculated for associations between variables.

\section{RESULTS}

Maturity score. Figure 1 depicts the frequency distribution of the gestational age of all cases as documented in the autopsy reports. Two maxima occur around 18 and $23 \mathrm{wk}$, in close relation to the first and second ultrasound screening dates. The gap between 30 and 32 wk possibly reflects the difficulties obtaining parental consent for the postmortem examination after a fatal clinical outcome of preterm infants of this gestational age. According to our refined histologic maturity score system, the human DA tissue specimens examined in our study were assigned to the following maturation stages: 26 specimens were stage I, 33 specimens were stage IIa, 11 specimens were IIb, and 10 specimens were stage III. None of our specimens had a closed lumen, meeting the histologic criteria of a stage IV DA. Gestational age and histologic maturity score corresponded in $66(83 \%)$ of 80 cases $\left(R^{2}=\right.$ $-0.81)$. Ten specimens were judged histologically more and four less mature than by estimated date. Figure $2 a-d$ shows a typical light micrograph for each maturity stage.

Of the 57 cases included for examination of COX and EP4 receptor expression, 20 specimens were maturity stage I, 22 were stage IIa, eight were stage IIb, and seven were stage III.

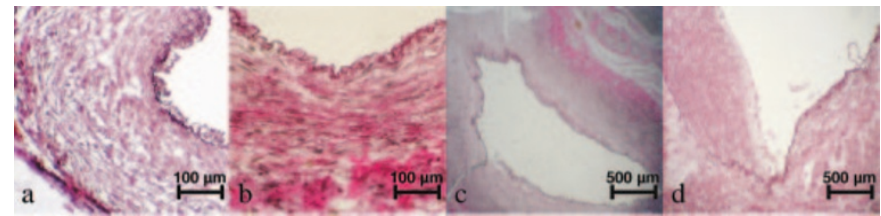

Figure 2. Elastica van Giesen staining of DA in different maturity stages. Light micrographs show DA maturity stages I (a), IIa (b), IIb (c), and III $(d)$. Although the DA in maturity stage I still displays a regular organ architecture, proliferative degeneration can be seen in stages IIb and III.

Although our results of the immunohistochemical examination of COX and EP4 receptor expression in the DA revealed no differences between the histologic maturity stages IIa and IIb, we refrained from combining the specimens into one group. Otherwise, we would have underestimated the significant morphologic differences between a DA just entering degenerative proliferation (stage IIa) and one in the middle of the process (stage IIb).

COX1-positive cells were found in all maturity stages from the 12th throughout the 20th week. COX1 staining intensity increased steadily during development in the endothelium, intima, and media and was strongest in maturity stage III (Fig. 3, upper row, endothelium $p<0.005$, intima $p<$ 0.0005 , media $p<0.0005$ stage III versus stage I). Throughout all maturity stages, COX1 staining intensity appeared strongest in the endothelium (Fig. $4 a$ and $b$ ). The cellular localization pattern of COX1 changed from a perinuclear staining pattern in stage I to a cytoplasmatic pattern in stage III (Fig. 4c).

Using a common staining protocol, no COX2-specific reaction was detectable in our specimens. Therefore, the catalyzed signal amplification method, which is about one order of magnitude more sensitive than standard detection systems, was applied. With this method, weak COX2 staining was detected in all three maturity stages. During development, a marginal increase in staining intensity was seen in maturity stages IIa and b, compared with almost no staining in stage III. 

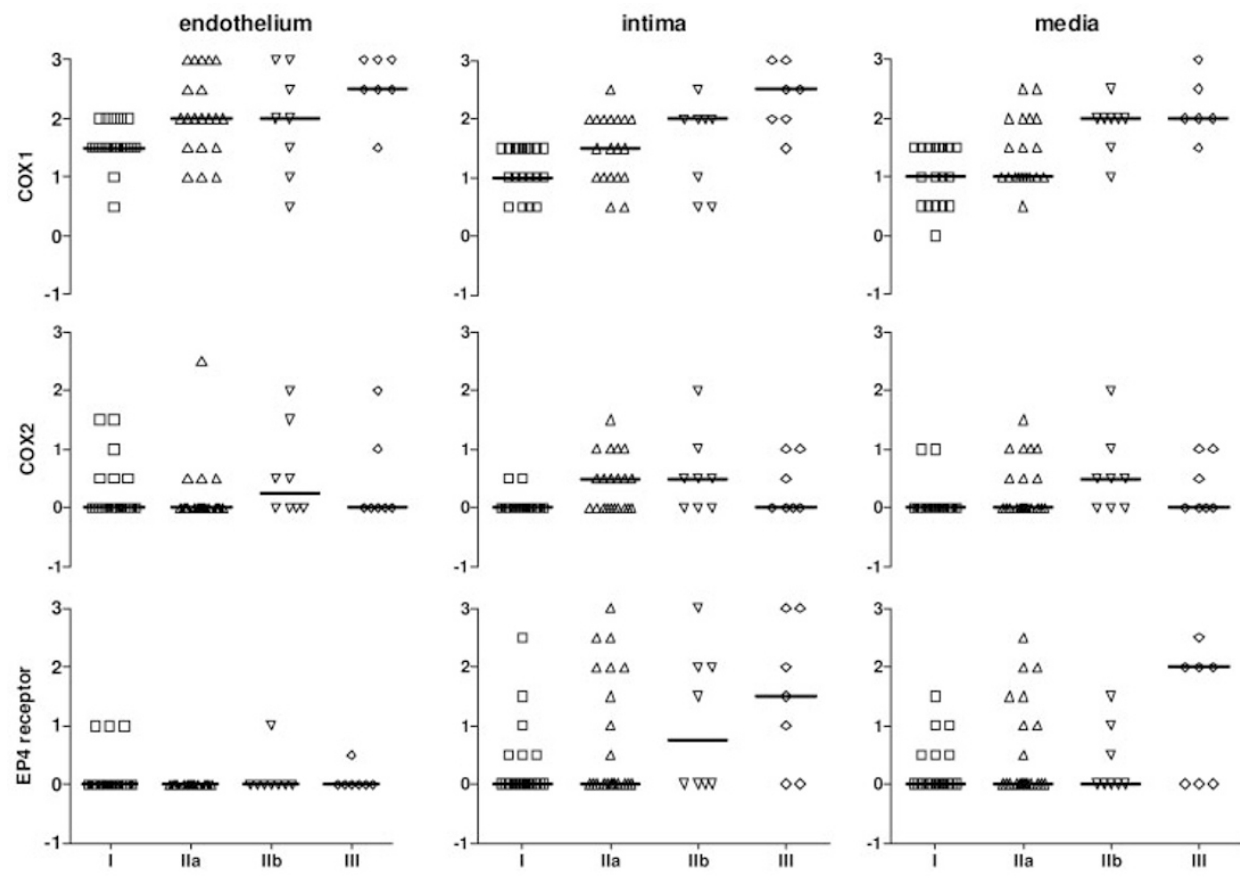

Figure 3. Maturity-dependent immunohistochemical staining intensity of COX1, COX2, and EP4 receptor in human DA. Semiquantitative evaluation of the staining intensity is expressed on a discrete scale ( $y$ axis) and separately scored for three vessel regions (left column, endothelium; middle column, intima; right column, media). The $x$ axis depicts maturity stages. Maturity stage I (squares) $n=20$, stage IIa (triangles pointing upward) $n=22$, stage $\mathrm{IIb}$ (triangles pointing downward) $n=8$, stage III (rhombi) $n=7$, complete data shown as dot plots and median. COX1 staining intensity (upper row) appeared strongest in the endothelium throughout all maturity stages (stage I endothelium $v s$ intima and media $p<0.005$, stage III not significant). COX2 staining intensity (middle row) remained weak during development. EP4 receptor staining intensity (lower row) was found especially in the intima in maturity stages IIb and III as well as in the media in stage III (stage III media $v s$ endothelium $p$ $<0.05)$
In contrast to $\mathrm{COX} 1$, the staining intensity of $\mathrm{COX} 2$ did not differ between endothelium, intima, and media (Fig. 3, middle row). COX2-positive staining was localized perinuclear and did not change localization throughout development. Specimens with a record of infection, which were excluded from COX evaluation, displayed an exceptionally strong expression of COX1 and 2 in all DA layers (data not shown).

EP4 receptor staining intensity increased moderately during development in the intima and media, but not as steady as the COX1 staining intensity. In the intima, EP4 receptor-positive staining was found throughout all maturity stages but especially in stages IIb and III, whereas medium positive staining intensity in the media rose only in maturity stage III (Fig. 3,

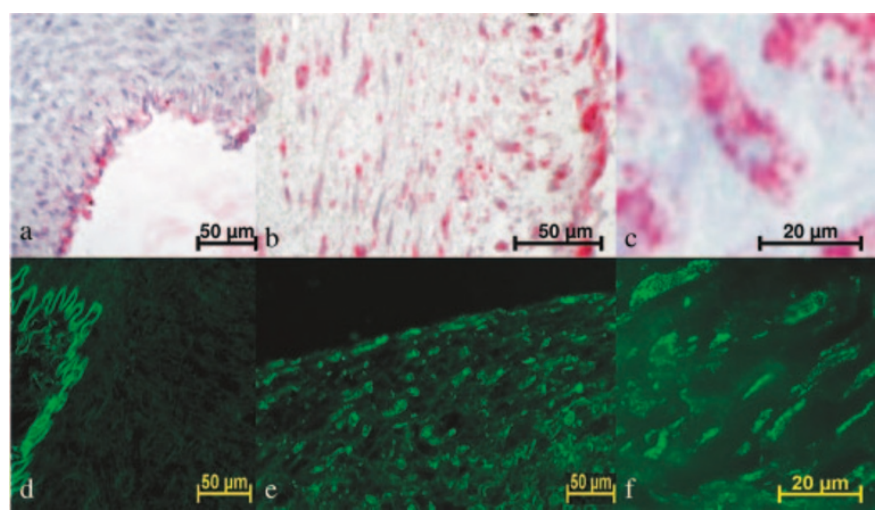

Figure 4. COX1 and EP4 immunostaining of DA in different maturity stages. COX1 immunostaining of DA maturity stages I $(a)$ and III $(b)$. COX1immunopositive cells stain red. In stage III, nearly all cells are COX1 positive. In both stages, endothelial staining is more intense than muscular staining. The detail of stage III (c) displays the cytoplasmic distribution of COX 1 . Fluorescence micrographs show EP4 receptor immunostaining of DA maturity stages I $(d)$ and III $(e)$. EP4 receptor-positive cells stain green. Although no EP4 staining was detected in stage I, the intima and media of the DA in stage III showed considerable staining intensity. The detail of stage III $(f)$ shows regular EP4 receptor distribution on the cell surface. lower row, Fig. $4 d$ and $e$ ). The endothelium never stained EP4 receptor immunopositive. Staining was regularly distributed on the cell surface (Fig. $4 f$ ).

COX1 and 2 staining in main arteries. Three to six sets of specimens of each maturity stage were examined. Weak COX1 staining was found in the main arteries. No staining differences were detected between the vessels, maturity stages, or vascular layers. No COX2 staining was found in the main arteries (data not shown). Evaluation of EP4 receptor staining intensity was not possible due to the strong background staining caused by the interaction of the secondary fluorescent antibody and the elastic fibers. No staining was found in negative controls regardless of gestational age, vessel type, or staining protocol.

\section{DISCUSSION}

Functional closure and remodeling of the DA are essential for postnatal adaptation. It is known that this complex alteration process already starts during gestation and involves several pathways, among which the PG metabolism seems to play a major role $(12,13)$. Until now, expression of the key factors of the PG metabolism, both COX isoforms and the EP4 receptor, has only been examined in fetal DA tissue from various animal species. Animal studies have not only revealed differences in the expression of these factors between species but also during the course of gestation (14-16). Based on this premise, we aimed to investigate whether both COX isoforms and the EP4 receptor express differently in the human DA during fetal development.

Research on human DA tissue is limited to the examination of postmortem specimens since cardiac surgery (e.g. ligation of a persistent DA) nearly never involves excision of viable DA tissue. Due to routine fixation procedures (e.g. formaldehyde), research methods are limited to immunohistochemical 
staining techniques, while molecular biology techniques require freshly processed tissue. Despite its methodologic limitations, immunohistochemistry is an established technique, widely used especially for routine diagnostic procedures.

In the present study, the large cohort of human fetal autopsy specimens were mostly derived from medically indicated abortions. Thus, influential effects on COX and EP4 receptor expression by exposure to oxygen during postnatal adaptation or postnatal pathology were avoided. Furthermore, specimens with documented vessel malformations or history of inflammation as a possible trigger for COX expression were excluded. The gestational age of our human fetal DA tissue specimens ranged from 11 to $38 \mathrm{wk}$, with two peaks in the frequency distribution around the dates of the first and second fetal ultrasound screening (Fig. 1). Histologic studies in the 1970s on human fetal and newborn DA tissue showed no strict relationship between gestational age and the stage of developmental maturity (3). We assumed that putative changes in COX expression are more closely connected to structural changes in the DA than to gestational age. To investigate the maturity-dependent protein expression of $\mathrm{COX} 1, \mathrm{COX} 2$, and EP4 receptor, we aimed to characterize the stage of histologic development in each of our specimens. For this purpose, we designed a ductal maturity score based on histologic studies by Gittenberger-de Groot et al. (2) and Desligneres and Larroche (3) and our own observations. The correlation of $R^{2}=0.81$ between the documented gestational age in the autopsy reports and our maturity score can be explained by either a limited reliability on the calculated gestational age or, more importantly, the above-discussed variation of fetal histologic DA maturation relative to gestational age. Among those 14 specimens with gestational age differing from the assigned histologic maturity score, 10 specimens were judged histologically more and four less mature than by estimated date. The 10 specimens histologically classified stage II rather than stage I had despite their lower gestational age already entered the proliferative degeneration phase, characterized by morphologic changes beginning around 20 wk of gestation.

Our study on fetal autopsy specimens demonstrated COX1 expression in all DA tissue regions that increased steadily during development and appeared strongest in the endothelium throughout all maturity stages (Fig. 2). In contrast to COX1, COX2 expression was weak in all three maturity stages, with no differences in staining intensity between the endothelium, intima, and media. These results confirm previous observations from a comparative study on COX1 and COX2 expression in normal and pathologic human tissue, which revealed ubiquitous COX1 expression including organ vasculature and DA, whereas no COX2 was detected in the latter. However, this study contained no data on source, number, or age of the examined DA specimens (17).

Our results of COX1 and 2 protein expression are consistent with studies carried out in the lamb and the pig, which showed that cyclooxygenases develop unevenly in the DA, with COX1 preceding COX2 in the course of gestation $(15,16)$. Besides considerable COX1 expression in muscle and endothelial cells from preterm and term lamb DA, Coceani et al. (18) only found a weak COX2 expression, which increased slightly toward the end of gestation. This finding corresponds to our observations of a marginal rise in the COX2 staining intensity around the start of the proliferative degeneration in maturity stages IIa and b. Nevertheless, this effect seemed to be transient because we detected almost no COX2 staining in the later developmental stage III. Our observation of a general increase of COX1 intensity during histologic maturation could be depicted in all tissue regions of the DA, seemingly without specific association with distinct morphologic changes during advanced maturation. A predominant perinuclear localization of both cyclooxygenases as reported by others in the murine DA (19) was only confirmed for COX2 throughout all developmental stages in our specimens, whereas we observed a change in the cellular localization pattern of COX1 from a perinuclear staining pattern in stage I to a cytoplasmic pattern in stage III. It remains speculative to assume a link between these changes in the cellular localization pattern of COX1 and the structural changes in the DA.

Although immunohistochemical studies alone allow no conclusion concerning activity and function of a protein, our findings on COX expression are in line with most studies in other mammalian species that assign the predominant role in the PG metabolism during gestation and in the premature to COX1. In contrast, the inducible isoform COX2 is thought to play an increasing role in PGE2 formation during the postnatal period. Clinical observations of frequently encountered reopening of a functionally closed DA during acute infection are in line with this scheme. Interestingly, we observed an exceptionally strong expression of COX2 in those specimens excluded from our study due to infection as well as in specimens of the umbilical artery, another vessel that closes during postnatal adaptation (data not shown). Furthermore, experiments in the lamb DA have confirmed a COX2 induction by triggers like endotoxin and oxygen (18). Results from knockout mouse models favor the idea that the two COX isoforms operate jointly around birth because COX1/COX2 double knockout mice die of PDA (20-22), whereas knockout of either COX does not affect ductal closure. Due to the small number of term specimens, we cannot contribute to the answer of this question.

Concerning the EP4 receptor protein expression, we observed a moderate increase of the staining intensity during development, limited to the intima and media. Comparative studies on porcine DA showed marked EP3 and EP4 receptor protein expression in the fetal but not in the newborn DA. This loss of PG receptors is viewed as the major mechanism promoting postnatal DA closure (23). Besides a comparative study on immature and mature fetal sheep and baboons, the only available study on EP4 receptor expression in a small number of human DA samples from neonates and infants undergoing DA surgery, showed markedly and unchanged EP4 receptor mRNA and protein expression (10). Furthermore, the reported changes in the susceptibility of PG receptors to PGE2 during gestation, resulting from alterations in the cAMP signaling pathway, might explain a decreased responsiveness of a term DA to PGE2 despite an unchanged receptor expression (24). Whether this is also applicable to the near- 
term human DA and what triggers this perinatal change of EP4 receptor susceptibility remain to be specified.

In conclusion, developmental variations of the DA are not closely related to gestational age and therefore a previous evaluation of the histologic DA maturity seems necessary for more exact results from immunohistochemical studies on protein expression of factors related to ductal development. Our findings confirm results from animal studies of different expression of the key players of the PG metabolism during the course of gestation. Although COX2 expression remained weak throughout development, COX1 and EP4 receptor expression rose steadily with advancing histologic maturity.

Further studies are necessary to elucidate the interaction between differential COX expression during fetal life and other essential factors preparing the DA for its postnatal definite closure.

\section{REFERENCES}

1. Bergwerff M, DeRuiter MC, Gittenberger-de Groot AC 1999 Comparative anatomy and ontogeny of the ductus arteriosus, a vascular outsider. Anat Embryol (Berl) 200:559-571

2. Gittenberger-de Groot AC, van Ertbruggen I, Moulaert AJ, Harinck E 1980 The ductus arteriosus in the preterm infant: histologic and clinical observations. J Pediatr 96:88-93

3. Desligneres S, Larroche JC 1970 Ductus arteriosus. I. Anatomical and histological study of its development during the second half of gestation and its closure after birth. II. Histological study of a few cases of patent ductus arteriosus in infancy. Biol Neonate 16:278-296

4. Reller MD, Lorenz JM, Kotagal UR, Meyer RA, Kaplan S 1985 Hemodynamically significant PDA: an echocardiographic and clinical assessment of incidence, natural history, and outcome in very low birth weight infants maintained in negative fluid balance. Pediatr Cardiol 6:17-23

5. Seidner SR, Chen YQ, Oprysko PR, Mauray F, Tse MM, Lin E, Koch C, Clyman RI 2001 Combined prostaglandin and nitric oxide inhibition produces anatomic remodeling and closure of the ductus arteriosus in the premature newborn baboon. Pediatr Res 50:365-373

6. Smith GC 1998 The pharmacology of the ductus arteriosus. Pharmacol Rev 50:35-58

7. Wright DH, Abran D, Bhattacharya M, Hou X, Bernier SG, Bouayad A, Fouron JC, Vazquez-Tello A, Beauchamp MH, Clyman RI, Peri K, Varma DR, Chemtob S 2001 Prostanoid receptors: ontogeny and implications in vascular physiology. Am J Physiol Regul Integr Comp Physiol 281:R1343-R1360

8. Smith GC, Coleman RA, McGrath JC 1994 Characterization of dilator prostanoid receptors in the fetal rabbit ductus arteriosus. J Pharmacol Exp Ther 271:390396
9. Momma K, Toyoshima K, Takeuchi D, Imamura S, Nakanishi T 2005 In vivo constriction of the fetal and neonatal ductus arteriosus by a prostanoid EP4-receptor antagonist in rats. Pediatr Res 58:971-975

10. Leonhardt A, Glaser A, Wegmann M, Schranz D, Seyberth H, Nusing R 2003 Expression of prostanoid receptors in human ductus arteriosus. $\mathrm{Br} \mathrm{J}$ Pharmacol 138:655-659

11. Denkert C, Winzer KJ, Muller BM, Weichert W, Pest S, Kobel M, Kristiansen G Reles A, Siegert A, Guski H, Hauptmann S 2003 Elevated expression of cyclooxygenase-2 is a negative prognostic factor for disease free survival and overall survival in patients with breast carcinoma. Cancer 97:2978-2987

12. Clyman RI 1987 Ductus arteriosus: current theories of prenatal and postnatal regulation. Semin Perinatol 11:64-71

13. Coceani F, Olley PM 1988 The control of cardiovascular shunts in the fetal and perinatal period. Can J Physiol Pharmacol 66:1129-1134

14. Bouayad A, Kajino H, Waleh N, Fouron JC, Andelfinger G, Varma DR, Skoll A, Vazquez A, Gobeil F Jr, Clyman RI, Chemtob S 2001 Characterization of PGE2 receptors in fetal and newborn lamb ductus arteriosus. Am J Physiol Heart Circ Physiol 280:H2342-H2349

15. Guerguerian AM, Hardy P, Bhattacharya M, Olley P, Clyman RI, Fouron JC, Chemtob S 1998 Expression of cyclooxygenases in ductus arteriosus of fetal and newborn pigs. Am J Obstet Gynecol 179:1618-1626

16. Takahashi Y, Roman C, Chemtob S, Tse MM, Lin E, Heymann MA, Clyman RI 2000 Cyclooxygenase-2 inhibitors constrict the fetal lamb ductus arteriosus both in vitro and in vivo. Am J Physiol Regul Integr Comp Physiol 278:R1496R1505

17. Koki A, Khan NK, Woerner BM, Dannenberg AJ, Olson L, Seibert K, Edwards D, Hardy M, Isakson P, Masferrer JL 2002 Cyclooxygenase-2 in human pathological disease. Adv Exp Med Biol 507:177-184

18. Coceani F, Ackerley C, Seidlitz E, Kelsey L 2001 Function of cyclo-oxygenase-1 and cyclo-oxygenase- 2 in the ductus arteriosus from foetal lamb: differential development and change by oxygen and endotoxin. Br J Pharmacol 132:241-251

19. Baragatti B, Brizzi F, Ackerley C, Barogi S, Ballou LR, Coceani F 2003 Cyclooxygenase-1 and cyclooxygenase- 2 in the mouse ductus arteriosus: individual activity and functional coupling with nitric oxide synthase. $\mathrm{Br} \mathrm{J}$ Pharmacol 139:1505-1515

20. Nguyen M, Camenisch T, Snouwaert JN, Hicks E, Coffman TM, Anderson PA, Malouf NN, Koller BH 1997 The prostaglandin receptor EP4 triggers remodelling of the cardiovascular system at birth. Nature 390:78-81

21. Segi E, Sugimoto Y, Yamasaki A, Aze Y, Oida H, Nishimura T, Murata T, Matsuoka T, Ushikubi F, Hirose M, Tanaka T, Yoshida N, Narumiya S, Ichikawa A 1998 Patent ductus arteriosus and neonatal death in prostaglandin receptor EP4-deficient mice. Biochem Biophys Res Commun 246:7-12

22. Loftin CD, Trivedi DB, Tiano HF, Clark JA, Lee CA, Epstein JA, Morham SG, Breyer MD, Nguyen M, Hawkins BM, Goulet JL, Smithies O, Koller BH, Langenbach R 2001 Failure of ductus arteriosus closure and remodeling in neonatal mice deficient in cyclooxygenase-1 and cyclooxygenase-2. Proc Natl Acad Sci U S A 98:1059-1064

23. Bhattacharya M, Asselin P, Hardy P, Guerguerian AM, Shichi H, Hou X, Varma DR, Bouayad A, Fouron JC, Clyman RI, Chemtob S 1999 Developmental changes in prostaglandin $\mathrm{E}(2)$ receptor subtypes in porcine ductus arteriosus. Possible contribution in altered responsiveness to prostaglandin $\mathrm{E}(2)$. Circulation 100:17511756

24. Waleh N, Kajino H, Marrache AM, Ginzinger D, Roman C, Seidner SR, Moss TJ, Fouron JC, Vazquez-Tello A, Chemtob S, Clyman RI 2004 Prostaglandin E2mediated relaxation of the ductus arteriosus: effects of gestational age on g proteincoupled receptor expression, signaling, and vasomotor control. Circulation 110:2326-2332 